\author{
An ASABE Meeting Presentation \\ DOI: https://doi.org/10.13031/iles.ILES18-073 \\ Paper Number: ILES18-073
}

2950 Niles Road, St. Joseph, MI 49085-9659, USA

269.429.0300 fax 269.429.3852 hq@asabe.org www.asabe.org

\title{
Application of an early warning to detect enteropathies in intensive broiler farming
}

\author{
Emanuela Tullo, Federica Borgonovo, Guido Grillia, Alessandra Micheletti, Giacomo Aletti,, \\ Susanna Lolli, Valentina Ferrante Marcella Guarino \\ Department of Environmental Science and Policy, Università degli Studi di Milano, Via Celoria, 10, 20133 \\ Milan, Italy \\ ${ }^{a}$ Department of Veterinary Medicine, Università degli Studi di Milano, Via Celoria, 10, 20133 Milan, Italy \\ Written for presentation at the \\ $10^{\text {th }}$ International Livestock Environment Symposium (ILES X) \\ Sponsored by ASABE \\ Omaha, Nebraska, USA \\ September 25-27, 2018
}

\begin{abstract}
Remote and wearable sensors can be combined with smart algorithms to continuously monitor a wide range of animal responses linked with stress, health status and welfare. The idea of real time monitoring assumes a simple way to measure variable that can give an early warning for the farmer providing clear and suitable alerts to help them in their routine. The prompt reaction to any change in health, welfare and productive status is the key for the reduction in drugs usage and for the improvement of animal wellbeing.

In intensive poultry farms, enteric disorders represent a major health issue; these pathologies could be multifactorial and are a major cause of performances reduction. Monitoring poultry health status takes a key role for management to reduce chemicals/drugs and their costs. Nowadays, the preventive use of antibiotics in intensive farming system is common and this practice could lead to the spreading of drugs in the environment, contributing to the phenomenon of antibiotic resistance. Due to the high priority of this issue, it is of great importance the early detection of any health problem in intensive farming. Precision Livestock Farming, through the combination of cheap technologies and specific algorithms, can provide valuable information for farmers starting from the huge amount of data collected in real time at farm level.

This study was aimed to the application of a PLF diagnostic tool, sensible to the variation of volatile organic compounds, to promptly recognize enteric problems in intensive farming, supporting veterinarians and enabling specific treatments in case of disease.
\end{abstract}

Keywords. early warning system, health problem, intensive farming, PLF, poultry farming, volatile organic compounds

\footnotetext{
The authors are solely responsible for the content of this meeting presentation. The presentation does not necessarily reflect the official position of the American Society of Agricultural and Biological Engineers (ASABE), and its printing and distribution does not constitute an endorsement of views which may be expressed. Meeting presentations are not subject to the formal peer review process by ASABE editorial committees; therefore, they are not to be presented as refereed publications. Publish your paper in our journal after successfully completing the peer review process. See www.asabe.org/JournalSubmission for details. Citation of this work should state that it is from an ASABE meeting paper. EXAMPLE: Author's Last Name, Initials. 2018. Title of presentation. ASABE Paper No. ---. St. Joseph, MI.: ASABE. For information about securing permission to reprint or reproduce a meeting presentation, please contact ASABE at www.asabe.org/permissions (2950 Niles Road, St. Joseph, MI 49085-9659 USA).
}

$10^{\text {th }}$ International Livestock Environment Symposium (ILES X) 


\section{Introduction}

Precision livestock farming (PLF) can be defined as real-time monitoring technologies aimed at managing the smallest manageable production unit’s temporal variability, known as ‘the per animal approach’(Halachmi \& Guarino, 2016).

Advances in engineering research and biomaterials, have resulted in the emergence of 'sensing solutions' and smart computing technologies for data collection and analysis. These technologies have the potential to address these problems by developing innovative diagnostic tools for the rapid detection of key health threats within the agri-food livestock sector.

The market for point-of-care testing in veterinary diagnostics is expected to increase at a compound annual growth rate (CAGR) of 18\%, reaching US\$6.71 Billion by 2021. Novel diagnostic tools and disease modelling will enable decisionmaking and investigate the rapid diagnosis of epidemic and emerging diseases of farmed animals (Neethirajan, Tuteja, Huang, \& Kelton, 2017).

In poultry farming, intestinal diseases have a major impact on technical performance and animal welfare. Decreased digestion efficacy and nutrients absorption exaggerated by an increased and changed need for energy and nutrients will lead to growth depression and increased feed conversion ratio (ter Veen, de Bruijn, Dijkman, \& de Wit, 2017).

Despite the importance of intestinal diseases, the determination of the causes is often difficult, because many infectious and non-infectious factors may influence intestinal health. A number of well-defined enteric pathogens and their pathology have been described. Of these Eimeria acervulina, E. maxima , E. tenella and necrotic enteritis are most important in broilers farming(ter Veen et al., 2017).

Today vaccinations can prevent few diseases, and enteric pathologies, unluckily, are not preventable and the diagnosis is available only when the pathology is full-blown. The broiler industry relies on in-feed prophylaxis with application of anticoccidial drugs (Haug, Gjevre, Thebo, Mattsson, \& Kaldhusdal, 2008), but this clashes with the public's concern regarding the use of drugs in intensive farming. Use of antimicrobials and anticoccidial drugs in animals poses a potential risk for public health since it contributes to the selection and spread of resistant microbes in the environment (Speksnijder, Mevius, Bruschke, \& Wagenaar, 2015) Antimicrobials resistance is the global health issue integrating human, animal, and environmental health that makes public bodies as UN or the WHO setting a global agenda to contrast the crisis(Laxminarayan, Sridhar, Blaser, Wang, \& Woolhouse, 2016). Even if substantial funds have been committed in the United States and Europe to tackle antimicrobials resistance, there is the need to incentivize the development of new vaccines, diagnostics, novel therapies, and stewardship methods (Laxminarayan et al., 2016).The clearest consequence of antibiotic release in natural environments is the selection of resistant bacteria (Martinez, 2009), causing antibiotic-resistant infections that add considerable costs to health care systems. Those infections require the use of antibiotics that are more toxic for the patient and frequently more expensive. Hospital stays became longer and patient can experience a higher incidence of long-term disability (Ventola, 2015).

Today, the diagnosis, usually expensive, needs two-three days to be confirmed, and this delay represent almost the $10 \%$ of a normal broiler meat production cycle (38-45 days), implying an economic loss for farmers. Thus, farmers need early and accurate diagnosis techniques of enteric infections, to treat quickly animals, and consequently, reduce the quantity and the costs of incorrect and long-term treatments.

In particular, diagnostic techniques must be rapid and sufficiently inexpensive if they aim to prevent the decision to start antibiotic treatment. Indeed, the application of specific diagnostics is important for carrying out rational and effective control measurements (McDougald \& Fitz-Coy, 2013).

The development of an alternative diagnostic tool might allow the prompt detection of the onset of infections. Several studies have explored the possibility of diagnosing pathologies in livestock and in humans via identification of Volatile Organic Compounds (VOCs) produced by pathogens, host-pathogen interactions and biochemical pathways (Guffanti, Pifferi, Falciola, \& Ferrante, 2017). VOCs are present in blood, breath, stool, sweat, skin, urine and vaginal fluids of humans and animals and their qualitative and quantitative composition is influenced by pathophysiological responses to infections, toxins or endogenous metabolic pathway perturbations (Ellis et al., 2014).

Odors in the barn are influenced by poultry health status and, in particular, enteric problems are characterized by peculiar odor properties (Sohn et al., 2008).

Precision livestock farming (PLF) can be defined as real-time monitoring technologies aimed at managing the smallest

manageable production unit’s temporal variability, known as 'the per animal approach'.

The goal of this study was the evaluation of the applicability of an early warning to detect enteropathies in intensive broiler farming.

\section{Material and methods}

An experimental trial and a validation trial were carried out in two poultry facilities located in the North of Italy. The experimental trial was performed in the experimental facilities of Università degli Studi di Milano (Lodi, Italy) and the validation trial performed in a commercial farm located nearby Brescia. Both trials lasted 45 day and Ross 308 chicks were 
used. Chicks in the experimental facilities were divided the first day in two separated pens (A and B, 2x3 m, bird stocking density $30 \mathrm{~kg} / \mathrm{m}^{2}$ ) with the same environmental conditions, the only difference was related to the addition of Robenidine (coccidiostatic) to the feed of group A. Also feed in the commercial facility (bird stocking density $33 \mathrm{~kg} / \mathrm{m}^{2}$ ) was added with the same active principle. During the experimental trial in each pen a weekly collection of faeces and air sample was performed to evaluate the possible presence of coccidia. Meanwhile, in the commercial barn only in the first, fourth and fifth week of the production cycle was possible to collect samples, due to an unexpected avian influenza outbreak that limited the access to the farm. In both trials faeces were collected in vials to perform the oocysts count in the laboratory of avian pathology, according to the Mc Master method (Holdsworth et al., 2004). The number of oocysts, expressed as oocysts/g of faeces (opg), was used as a Gold Standard (Tullo et al., 2017) to indicate the health status of broiler (infected/not infected) and was used as a reference compared to the VOCs analysis performed on air samples. Simultaneously to faeces collections, air sampling was carried out following the recommendations described in the European Standard EN 13725 (CEN, 2003). Air was drawn into disposable Nalophan ${ }^{\circledR}$ bags, using a special sampler that works according to the lung principle. The bags containing air from the poultry facilities were transported to the laboratory and analyzed within $1 \mathrm{~h}$ after sampling with a prototype based on a patented technology (International Publication Number: WO2017/212437). The prototype was developed to draw and to analyze the air samples, giving a response on VOCs present in the air.

Data obtained on air samples collected in the experimental facility were processed using multivariate statistical techniques, specifically Principal Component Analysis (PCA) and Linear Discriminant Analysis (LDA). Discrimination powers (DPs), which are an inverse measure of the overlapping of two groups of measurement points, between pen $A$ and B was also calculated along the PCA (Bro \& Smilde, 2014). A correlation classifier statistical procedure (K nearest neighbors, KNN) was then performed to validate LDA results. K nearest neighbors is a simple algorithm that stores all available measurements and classifies new measurements based on a similarity criterion (e.g., distance functions). A measurement is classified by a majority vote of its neighbors, with the new measure being assigned to the class most common amongst its $\mathrm{K}$ nearest neighbors evaluated by a distance function. Measurements from the commercial farm were classified according to the KNN, with $\mathrm{K}$ equal to 3 . As it is, the new samples were classified according to similarity with at least 3 reference measurements from the experimental trial.

\section{Results and Discussion}

During the experimental trial, only pen B developed coccidiosis, associated with clinical signs (diarrhoea), and the oocyst count reached the value of 37,300 opg at week 4. Consequently, pen A and B were defined as not infected and infected, respectively.

According to the classification based on oocysts count, PCA showed good ability in the discrimination between air samples from pen A and pen B. PCA were calculated between pens for each week and reported in Figure 1.

PCA showed good ability to discriminate each week between air samples from pen A (not infected) and pen B (infected).
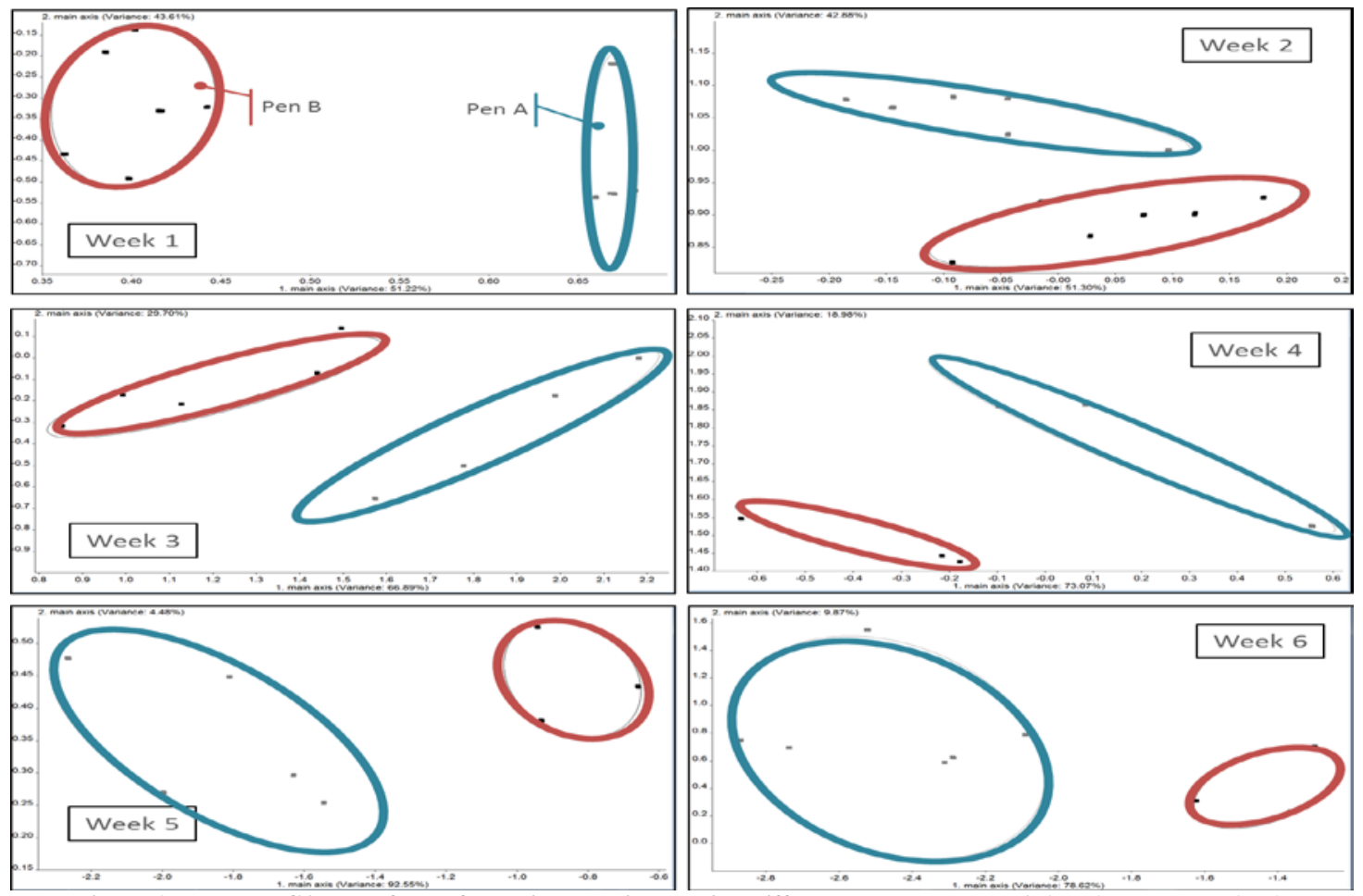

Figure 1. Weekly PCA plot of data from air analysis, showing differences between pen A (blue) and pen B (red). 
Discrimination powers (DPs) along the PCA were calculated between pens for each week and reported in Figure 2 The value was high (0.95) even in the first week when the infection in pen B was at a very early stage (only 250 opg, as shown), and this could set the basis for the development of a new device able to rapidly detect the onset of coccidiosis in poultry farms.

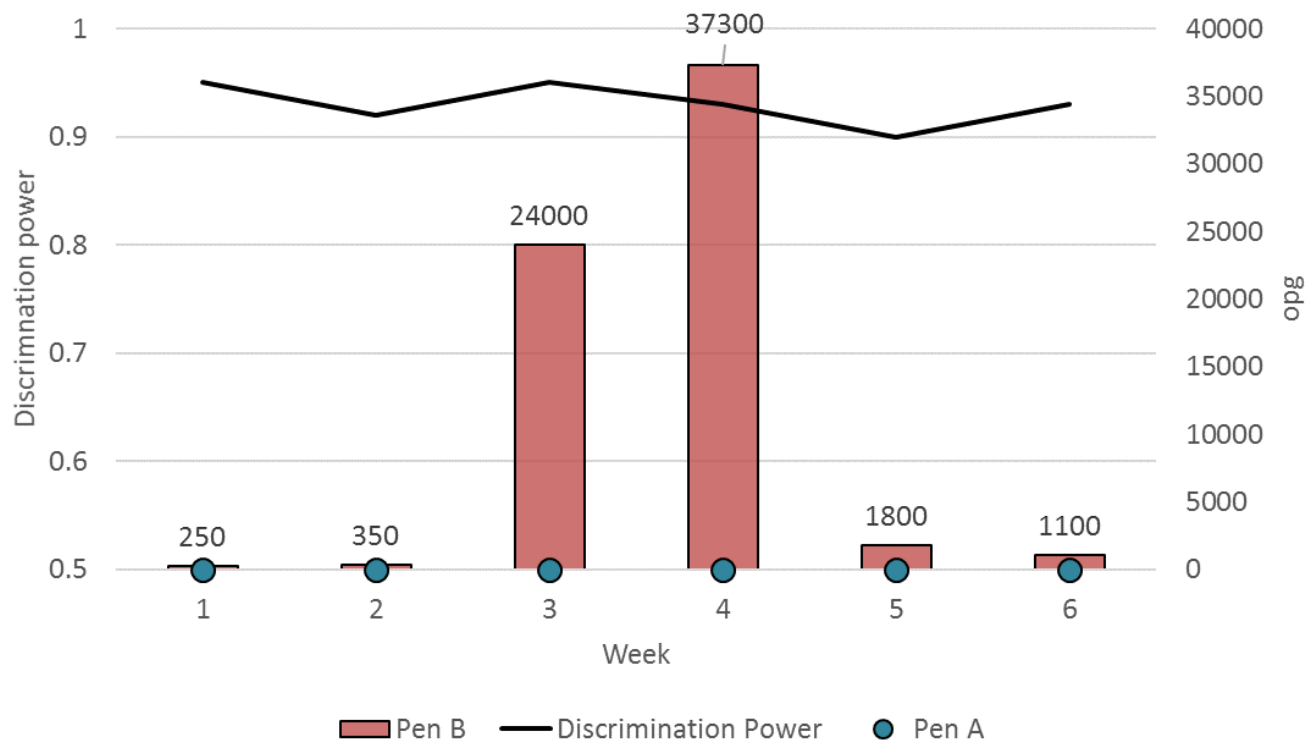

Figure 2. Discrimination power (DP) and oocysts count for pen A and B during the experimental trial

Since PCA has shown differences between air samples from infected and not infected animals, classes were modelled using Linear Discriminant Analysis which showed that the difference among groups was relevant starting from the first week, confirming PCA findings. The information about the presence of the infection is captured by the first component, which explains $48.5 \%$ of the total variance.

In order to validate whether the prototype was able to early identify the infection in an intensive poultry farm, the $\mathrm{K}$ Nearest Neighbours (KNN) classification technique, performed on results of LDA, was carried out on data from the commercial farm, using data collected in the experimental trial as reference dataset.

Data collected in the first week, in the commercial farm, were classified as "not infected" and this result was validated by the Gold Standard that confirmed the absence of infection (0 opg).

Meanwhile, data collected at the fourth and fifth week, in the commercial farm, were classified as "infected". These results were validated by the oocyst count that was 50,000 opg at week four (Figure 3).

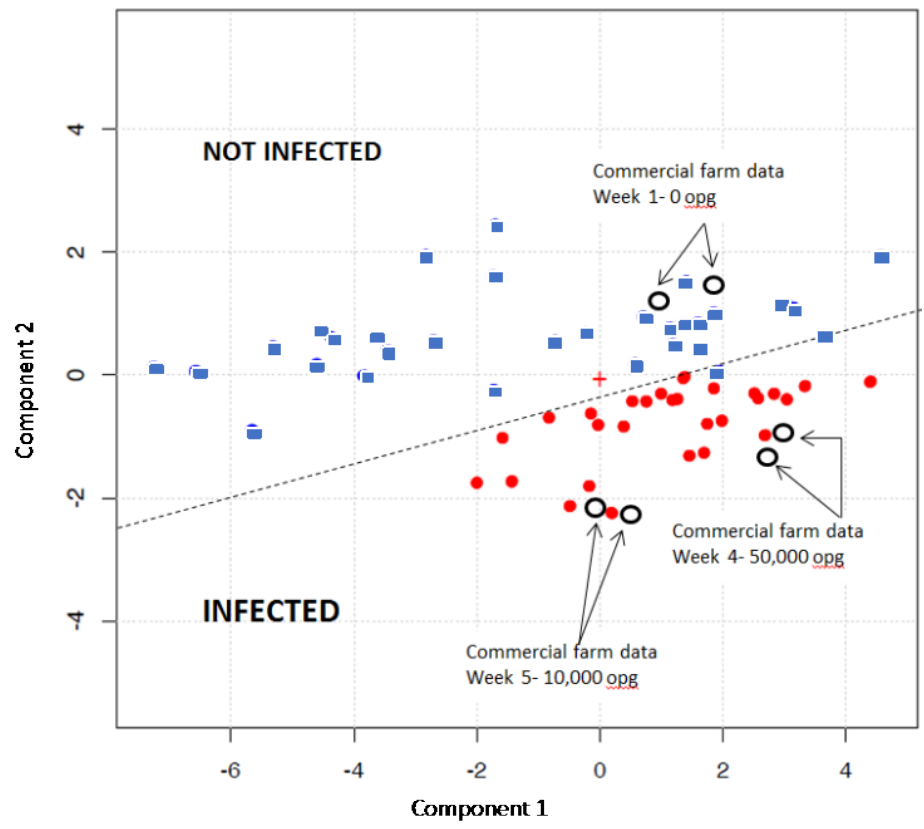

Figure 3. Validation of the prototype using data from commercial farm 


\section{Conclusion}

This pilot study has shown that the prototype perfectly suits the methodologies and the goals of Precision Livestock Farming (PLF), which consists of non-invasive automated technologies that can support farmers with early warning systems for the identification of production, health and welfare problems on farms (Tullo et al., 2017). Indeed, the prototype was able to discriminate among VOCs emitted by animals infected by coccidiosis and the discrimination was effective even when the infection was at a very early stage (only $250 \mathrm{opg}$ ).

Nowadays, the use of Smart Networked Objects and Internet of Things, opened a new era of communication in which things, humans and animals are part of a data network exchange, leading to a new concept of farming. Remote and wearable sensors can be combined with smart algorithms to continuously monitor a wide range of animal responses. Real-time monitoring is a simple way to measure variables providing a signal of early warning to the farmer.

The prompt reaction to any change in health, welfare and productive status is the key for the reduction of drugs usage and for the improvement of animal wellbeing. For this reason, the application of our prototype in livestock farming could be extremely beneficial

\section{References}

Bro, R., \& Smilde, A. K. (2014). Principal component analysis. Analytical Methods, 6(9), 2812-2831.

Ellis, C. K., Stahl, R. S., Nol, P., Waters, W. R., Palmer, M. V., Rhyan, J. C., . . Salman, M. D. (2014). A Pilot Study Exploring the Use of Breath Analysis to Differentiate Healthy Cattle from Cattle Experimentally Infected with Mycobacterium bovis. PloS one, 9(2), e89280. doi:10.1371/journal.pone.0089280

Guffanti, P., Pifferi, V., Falciola, L., \& Ferrante, V. (2017). Analyses of odours from concentrated animal feeding operations: A review. Atmospheric Environment.

Halachmi, I., \& Guarino, M. (2016). Editorial: Precision livestock farming: a 'per animal' approach using advanced monitoring technologies. Animal, 10(9), 1482-1483. doi:10.1017/s1751731116001142

Haug, A., Gjevre, A.-G., Thebo, P., Mattsson, J. G., \& Kaldhusdal, M. (2008). Coccidial infections in commercial broilers: epidemiological aspects and comparison of Eimeria species identification by morphometric and polymerase chain reaction techniques. Avian pathology, 37(2), 161-170.

Holdsworth, P., Conway, D., McKenzie, M., Dayton, A., Chapman, H., Mathis, G., . . . Williams, R. (2004). World Association for the Advancement of Veterinary Parasitology (WAAVP) guidelines for evaluating the efficacy of anticoccidial drugs in chickens and turkeys. Veterinary parasitology, 121(3-4), 189-212.

Laxminarayan, R., Sridhar, D., Blaser, M., Wang, M., \& Woolhouse, M. (2016). Achieving global targets for antimicrobial resistance. Science, 353(6302), 874-875.

Martinez, J. L. (2009). Environmental pollution by antibiotics and by antibiotic resistance determinants. Environ Pollut, 157(11), 2893-2902. doi:10.1016/j.envpol.2009.05.051

McDougald, L., \& Fitz-Coy, S. (2013). Coccidiosis. In Disease of Poultry (13th ed., pp. 1148-1163): Iowa State Press Ames.

Neethirajan, S., Tuteja, S. K., Huang, S.-T., \& Kelton, D. (2017). Recent advancement in biosensors technology for animal and livestock health management. Biosensors and Bioelectronics, 98(Supplement C), 398-407. doi:https://doi.org/10.1016/j.bios.2017.07.015

Sohn, J. H., Hudson, N., Gallagher, E., Dunlop, M., Zeller, L., \& Atzeni, M. (2008). Implementation of an electronic nose for continuous odour monitoring in a poultry shed. Sensors and Actuators B: Chemical, 133(1), 60-69.

Speksnijder, D., Mevius, D., Bruschke, C., \& Wagenaar, J. (2015). Reduction of veterinary antimicrobial use in the Netherlands. The Dutch success model. Zoonoses and public health, 62, 79-87.

ter Veen, C., de Bruijn, N. D., Dijkman, R., \& de Wit, J. J. (2017). Prevalence of histopathological intestinal lesions and enteric pathogens in Dutch commercial broilers with time. Avian Pathology, 46(1), 95-105. doi:10.1080/03079457.2016.1223271

Tullo, E., Fontana, I., Diana, A., Norton, T., Berckmans, D., \& Guarino, M. (2017). Application note: Labelling, a methodology to develop reliable algorithm in PLF. Computers and Electronics in Agriculture, 142, 424-428.

Ventola, C. L. (2015). The antibiotic resistance crisis: part 1: causes and threats. P T, 40(4), 277-283. 